\title{
SYMMETRIC DETERMINANTS AND JORDAN NORM SIMILARITIES IN CHARACTERISTIC 2
}

\author{
WILLIAM C. WATERHOUSE ${ }^{1}$
}

\begin{abstract}
We first determine all linear changes of variable formally preserving symmetric determinants in characteristic 2 ; there are just slightly more of them than in other characteristics. We then restate this result in terms of affine group schemes. This allows us to apply descent theory, and thereby we prove a theorem on norm similarities of Jordan algebras in the one case left open by Jacobson.
\end{abstract}

Consider an $n \times n$ symmetric matrix with indeterminate entries $X_{i}$ on the diagonal and $Y_{i j}$ (for $\left.i<j\right)$ in the $(i, j)$ and $(j, i)$ positions. Let $D(X, Y)$ be its determinant. We are interested in the linear changes of variable (in $X$ and $Y$ ), with coefficients in a fixed commutative ring $R$, that take $D$ to a constant multiple of itself. When 2 is not a zero-divisor in $R$, these maps are known [5, §6]. In this paper we will consider only the case where $R$ has characteristic 2 .

Once the characteristic is 2 , we can evaluate $D$ by the classical formula [4, p. 410] for a determinant with skew off-diagonal entries:

$$
D=\sum_{I} \operatorname{Pf}_{I}\left(Y_{i j}\right)^{2} \cdot \prod_{i \notin I} X_{i}
$$

where $\mathrm{Pf}_{I}$ means the Pfaffian of the alternating matrix having entries $Y_{i j}$ with $i, j$ in I. The sum runs over all subsets $I$ of $\{1,2, \ldots, n\}$ containing an even number of elements; it includes $I=\varnothing$, with the Pfaffian put equal to 1 . Indeed, if we expand out a determinant and look at the terms containing just a specified set of diagonal entries, we find that the remaining factors in those terms are the diagonal-avoiding terms in the complementary principal minor (an observation due to Cayley). In our case the off-diagonal entries are skew, and so these remaining factors add up to the square of the Pfaffian (giving zero, of course, for odd sizes). As $\mathrm{Pf}_{I}$ is a polynomial with coefficients in the prime field $\mathbf{F}_{2}$, we can move the square inside and write

$$
D=\sum_{I} \operatorname{Pf}_{I}\left(Y_{i j}^{2}\right) \cdot \prod_{i \notin I} X_{i}
$$

Received by the editors July 13, 1984 and, in revised form, August 27, 1984.

1980 Mathematics Subject Classification. Primary 15A15, 17C20; Secondary 14L15.

${ }^{1}$ This work was supported in part by the NSF Grants MCS 8102697 and DMS 8400649. 


\section{A determinant identity.}

LEMMA 1. Let $\left(a_{i}^{j}\right)$ be elements in a ring of characteristic 2 , with $1 \leqslant i, j \leqslant n$. Then

$$
\begin{gathered}
\sum_{I} \operatorname{Pf}_{I}\left(\sum_{k} a_{i}^{k} a_{j}^{k} X_{k}^{2}+\sum_{r<s}\left(a_{i}^{r} a_{j}^{s}+a_{i}^{s} a_{j}^{r}\right) Y_{r s}^{2}\right) \cdot \prod_{i \notin I}\left(\sum_{j} a_{i}^{j} X_{j}\right) \\
=\operatorname{det}\left(a_{i}^{j}\right) \cdot D(X, Y) .
\end{gathered}
$$

Proof. It is enough to prove this identity for $a_{i}^{j}$ indeterminates over $\mathbf{F}_{2}$. Extend the ring $\mathbf{F}_{2}\left[\left(a_{i}^{j}\right)\right]$ by adjoining square roots $p_{i}^{j}$, so $a_{i}^{j}=\left(p_{i}^{j}\right)^{2}$. Let $P$ be the matrix $\left(p_{i}^{j}\right)$. The map replacing our original $X, Y$ matrix by

$$
P\left(\begin{array}{lll}
X_{1} & Y_{12} & \cdots \\
\vdots & & \vdots \\
\cdots & \cdots & \cdots
\end{array}\right) P^{\mathrm{tr}}
$$

multiplies the determinant by $(\operatorname{det} P)^{2}=\operatorname{det}\left(a_{i}^{j}\right)$. But it is straightforward to compute that this map replaces the diagonal entry $X_{i}$ by $\sum_{j}\left(p_{i}^{j}\right)^{2} X_{j}$, or $\sum_{j} a_{i}^{j} X_{j}$, while it replaces the off-diagonal entry $Y_{i j}$ by

$$
\sum_{k} p_{i}^{k} p_{j}^{k} X_{k}+\sum_{r<s}\left(p_{i}^{r} p_{j}^{s}+p_{i}^{s} p_{j}^{r}\right) Y_{r s} .
$$

As the characteristic is 2 , the square of this expression is

$$
\sum_{k} a_{i}^{k} a_{j}^{k} X_{k}^{2}+\sum_{r<s}\left(a_{i}^{r} a_{j}^{s}+a_{i}^{s} a_{j}^{r}\right) Y_{r s}^{2}
$$

Thus the formula for $D$, applied to our new determinant, does yield the left side of the identity stated in the lemma.

\section{Changes of variable perserving $D(X, Y)$.}

THEOREM 2. Let

$$
U_{i}=\sum a_{i}^{j} X_{j}+\sum b_{i}^{j k} Y_{j k} \quad \text { and } \quad V_{i j}=\sum c_{i j}^{k} X_{k}+\sum d_{i j}^{r s} Y_{r s},
$$

where the coefficients lie in a ring of characteristic 2. These expressions will be new independent variables with $D(U, V)$ a constant multiple of $D(X, Y)$ precisely when:

(1) $\operatorname{det}\left(a_{i}^{j}\right)$ is invertible,

(2) all $b_{i}^{j k}=0$,

(3) $\left(c_{i j}^{k}\right)^{2}=a_{i}^{k} a_{j}^{k}$, and

(4) $\left(d_{i j}^{r s}\right)^{2}=a_{i}^{r} a_{j}^{s}+a_{i}^{s} a_{j}^{r}$.

Under these conditions, $D(U, V)=\operatorname{det}\left(a_{i}^{j}\right) D(X, Y)$.

Proof. It follows from Lemma 1 that the conditions are sufficient. Indeed, only $\left(V_{i j}\right)^{2}$ enters the formula for $D(U, V)$, and conditions (3) and (4) allow us to express the coefficients in $\left(V_{i j}\right)^{2}$ in terms of the $a_{r}^{s}$. Since condition (2) shows that no $Y_{j k}$ terms occur in $U_{i}$, we have $D(U, V)=\operatorname{det}\left(a_{i}^{j}\right) D(X, Y)$ by the lemma.

Suppose now, conversely, that we have an arbitrary change of variables for which

$$
D(U, V)=\sum_{I} \operatorname{Pf}_{I}\left(V_{i j}^{2}\right) \cdot \prod_{i \notin I} U_{i}
$$


is a constant multiple $\alpha$ times $D(X, Y)$. We assume, of course, that the change of variables is invertible, so the $U_{i}$ and $V_{i j}$ are again independent variables. To analyze the identity $D(U(X, Y), V(X, Y))=\alpha D(X, Y)$, we can use a method due originally (I believe) to Frobenius [1]. What we do is to introduce a new variable $Z$, add it to one of $X_{i}$ or $Y_{i j}$, expand the resulting identity, and compare the terms of the same degree in $Z$.

First, let us add $Z$ to $Y_{p q}$. In $D(X, Y)$, the variable $Y_{p q}$ occurs only as $Y_{p q}^{2}$. Thus there will be no terms of degree 1 in $Z$. Similarly, $D(U, V)$ will not give any terms of degree 1 in $Z$ arising from the $Y_{p q}$ in $\operatorname{Pf}_{I}\left(V_{i j}^{2}\right)$. Thus the only terms of degree 1 in $Z$ are those occuring in

$$
\sum_{I} \operatorname{Pf}_{I}\left(V_{i j}^{2}\right) \cdot \prod_{i \notin I}\left(U_{i}+b_{i}^{p q} Z\right)
$$

namely,

$$
\sum_{I} \operatorname{Pf}_{I}\left(V_{i j}^{2}\right) \cdot \sum_{r \notin I}\left(\prod_{\substack{j \notin I \\ j \neq r}} U_{j}\right) b_{r}^{p q} Z .
$$

The coefficient of $Z$ in this sum must therefore be identically zero. In particular, looking only at the terms involving no $V_{i j}^{2}$, we find that

$$
\sum_{r}\left(\prod_{j \notin r} U_{j}\right) b_{r}^{p q}
$$

must be identically zero. As the $U_{j}$ are independent variables, this implies condition (2) of the theorem.

At this point we might look back at the identity $D(U, V)=\alpha D(X, Y)$. The term $\alpha X_{1} \cdots X_{n}$ on the right can arise only from the term $U_{1} \cdots U_{n}$ on the left, since all other terms there involve squares. But the coefficient of $X_{1} \cdots X_{n}$ in $U_{1} \cdots U_{n}$ is just $\operatorname{det}\left(a_{i}^{j}\right)$, since the characteristic is 2 and signs are irrelevant in the determinant. As our change of variables can be inverted, $\alpha$ is invertible, and thus condition (1) must hold.

Continuing with the identity $D(U, V)=\alpha D(X, Y)$, let us look for terms of the form $Y_{p q}^{2}\left(\Pi_{i \neq p, q} X_{i}\right)$. Such a term occurs exactly once in $D(X, Y)$. In $D(U, V)$, no such monomial will occur for any $I$ containing 4 or more indices, since each term in $\operatorname{Pf}_{I}\left(V^{2}\right)$ either will have a higher degree in $Y$ or will involve the square of some $X_{i}$. By condition (2), no terms of our form occur in $U_{1} \cdots U_{n}$. Thus such terms arise only from subsets $I$ containing exactly two indices. These subsets give

$$
\sum_{r<s}\left(d_{p q}^{r s}\right)^{2} Y_{p q}^{2} \cdot \prod_{j \neq r, s} U_{j}
$$

Now the coefficient of $\prod_{i \neq u, v} X_{i}$ in $\prod_{j \neq r, s} U_{j}$ is simply an $(n-2) \times(n-2)$ minor determinant of $\left(a_{i}^{j}\right)$, since again signs are irrelevant in characteristic 2 . Let us denote this minor by $M_{u v}^{r s}$. We know that $D(X, Y)$ does not contain any term with $Y_{p q}^{2}$ times any other product of $X$ 's. Thus

$$
\sum_{r<s}\left(d_{p q}^{r s}\right)^{2} \cdot M_{u v}^{r s}= \begin{cases}\alpha=\operatorname{det}\left(a_{i}^{j}\right) & \text { if } p=u, q=v \\ 0 & \text { otherwise }\end{cases}
$$


As an equation on square matrices of size $n(n-1) / 2$, this says that

$$
\left[\left(d_{p q}^{r s}\right)^{2}\right] \cdot\left[\wedge^{n-2}\left(a_{i}^{j}\right)\right]=\operatorname{det}\left(a_{i}^{j}\right) \cdot I d .
$$

But there is a classical identity on adjoints, and once we ignore signs it tells us that

$$
\left[\wedge^{2}\left(a_{i}^{j}\right)\right] \cdot\left[\wedge^{n-2}\left(a_{i}^{j}\right)\right]=\operatorname{det}\left(a_{i}^{j}\right) \cdot I d .
$$

Since we already know $\operatorname{det}\left(a_{i}^{j}\right)$ is invertible, we know that $\wedge^{n-2}\left(a_{i}^{j}\right)$ is an invertible matrix, and hence we can deduce

$$
\left[\left(d_{p q}^{r s}\right)^{2}\right]=\left[\wedge^{2}\left(a_{i}^{j}\right)\right]
$$

When we read this off entry by entry, we get condition (4).

Finally, to determine the $c_{i j}^{k}$ we consider the effect of changing $X_{k}$ to $X_{k}+Z$. In $D(X, Y)$, the factor $X_{k}$ occurs only to the first power, and thus we will get no terms at all that involve $Z^{2}$. Hence when we make the corresponding change in $U$ and $V$, the $Z^{2}$-terms must all cancel. The expression we have, to begin with, is

$$
\sum_{I} \operatorname{Pf}_{I}\left(V_{i j}^{2}+\left(c_{i j}^{k} Z\right)^{2}\right) \cdot \prod_{i \notin I}\left(U_{i}+a_{i}^{k} Z\right) \text {. }
$$

Let us focus on the terms that involve $Z^{2}$ and $(n-2)$ distinct $U_{i}$, with no $V_{i j}$ factors or higher $U_{i}$-powers. As in the previous argument, we see that such terms can come only from $I$ with less than 4 elements. Those with 2 elements give

$$
\sum_{i<j}\left(c_{i j}^{k}\right)^{2} Z^{2} \cdot \prod_{r \neq i, j} U_{r}
$$

while $I=\varnothing$ gives

$$
\sum_{i<j} a_{i}^{k} a_{j}^{k} Z^{2} \cdot \prod_{r \neq i, j} U_{r}
$$

The $U_{r}$ are independent, and these terms must cancel each other; thus condition (3) must hold.

3. Interpretation using affine group schemes. The conditions in Theorem 2 define an affine group scheme $F$ over $\mathbf{F}_{2}$. That is [5], for every $R$ of characteristic 2 they single out a subgroup $F(R)$ inside $\mathrm{GL}_{n(n+1) / 2}(R)$, and this subgroup is defined by polynomial equations on the matrix entries. Our goal now is to analyze this affine group scheme. One result will be a more explicit determination of $F(R)$ when $R$ is reduced.

Proposition 3. The Lie algebra $\operatorname{Lie}(F)$ has dimension $1+n^{2}\left(n^{2}-1\right) / 4$.

Proof. Let $R=\mathrm{F}_{2}[\varepsilon]$ with $\varepsilon^{2}=0$. The Lie algebra of a group scheme, in one of its equivalent forms [5, Chapter 12], consists of those elements in $F(R)$ that reduce to the identity when we reduce $\varepsilon$ to 0 . Condition (2) tells us that the $b_{i}^{j k}$ are zero. The other entries will have the form

$$
a_{i}^{j}=\delta_{i}^{j}+\varepsilon A_{i}^{j}, \quad c_{i j}^{k}=\varepsilon C_{i j}^{k}, \quad d_{i j}^{r s}=\delta_{i}^{r} \delta_{j}^{s}+\varepsilon D_{i j}^{r s}
$$


for scalars $A, C, D$. The invertibility of $\operatorname{det}\left(a_{i}^{j}\right)$ is automatic for such elements. Condition (3) requires $\left(c_{i j}^{k}\right)^{2}$, which is $=\varepsilon^{2}\left(C_{i j}^{k}\right)^{2}=0$, to equal

$$
a_{i}^{k} a_{j}^{k}=\varepsilon\left(\delta_{i}^{k} A_{j}^{k}+\delta_{j}^{k} A_{i}^{k}\right) .
$$

This imposes no restrictions on the $c_{i j}^{k}$, but it forces $\delta_{i}^{k} A_{j}^{k}+\delta_{j}^{k} A_{i}^{k}=0$. This says that $A_{p}^{q}=0$ for $p \neq q$.

Finally, condition (4) deals with $\left(d_{i j}^{r s}\right)^{2}$, which $=\left(\delta_{i}^{r} \delta_{j}^{s}+\varepsilon D_{i j}^{r s}\right)^{2}=\delta_{i}^{r} \delta_{j}^{s}$; this must equal $a_{i}^{r} a_{j}^{s}+a_{i}^{s} a_{j}^{r}$. Since $A_{p}^{q}=0$ for $p \neq q$, this condition is automatic unless $i=r$ and $j=s$; in that case, it gives us $1=1+\varepsilon\left(A_{i}^{i}+A_{j}^{j}\right)$. Thus all $A_{i}^{i}$ are equal.

All in all, then, we have one degree of freedom among the $A_{i}^{j}$ and free choice of the $n \cdot n(n-1) / 2$ different $C_{i j}^{k}$ and the $[n(n-1) / 2]^{2}$ different $D_{i j}^{r s}$. These dimensions add up to $1+n^{2}\left(n^{2}-1\right) / 4$.

In characteristic zero, the dimension of the Lie algebra equals the dimension of the group. In positive characteristics, this is true only for "smooth" group schemes. In characteristic $\neq 2$, the group scheme preserving symmetric determinants up to scalar is, in fact, still smooth. (This was first stated explicitly in [6,7], but it is an immediate consequence of results proved earlier by Jacobson [2, 3].) Here in characteristic 2, however, we shall see that for $n>2$ the group scheme $F$ is not smooth. To show this, we use two maps interrelating $F$ and $\mathrm{GL}_{n}$.

First of all, the computation in the proof of Lemma 1 shows that there is a homomorphism $\mathrm{GL}_{n}(R) \rightarrow F(R)$ sending $P$ to $P(\quad) P^{\mathrm{tr}}$. The kernel of this map is easily computed: it is $\mu_{2}(R)$, the scalars $\alpha$ with $\alpha^{2}=1$. (There may be nontrivial such $\alpha$ even in characteristic 2 , since $R$ in general is not assumed to be a field.) Group schemes like this kernel, trivial on fields, may be called "infinitesimal".

The formulas in Theorem 2 show that there is also a natural homomorphism $F(R) \rightarrow \mathrm{GL}_{n}(R)$, sending an element in $F(R)$ to $\left(a_{i}^{j}\right)$. The kernel of this map is again infinitesimal: for when $a_{i}^{j}=\delta_{i}^{j}$, we have $\left(c_{i j}^{k}\right)^{2}=0$ and $\left(d_{i j}^{r s}\right)^{2}=\delta_{i}^{r} \delta_{j}^{s}$, and these conditions force an element in $F(R)$ to be the identity when $R$ is a field. Finally, the computations in Lemma 1 show that the composite map $\mathrm{GL}_{n} \rightarrow F \rightarrow$ $\mathrm{GL}_{n}$ sends $\left(p_{i}^{j}\right)$ to $\left(\left[p_{i}^{j}\right]^{2}\right)$. This is what is called the Frobenius endomorphism in characteristic 2, and on smooth groups like $\mathrm{GL}_{n}$ it is an epimorphism (surjective when $R$ is an algebraically closed field). Hence $F \rightarrow \mathrm{GL}_{n}$ is an epimorphism. Since the infinitestimal kernel is connected of dimension 0 , and $\mathrm{GL}_{n}$ is connected of dimension $n^{2}$, we have

Proposition 4. The group scheme $F$ is connected and has dimension $n^{2}$.

Comparing this with the result in Proposition 3, we immediately have

COROLlARY 5. If $n \geqslant 3$, the group scheme $F$ is not smooth.

Let $A$ be the algebra over $\mathbf{F}_{2}$ constructed by taking the formal ring

$$
\mathbf{F}_{2}\left[a_{i}^{j}, c_{i j}^{k}, d_{i j}^{r s}, 1 / \operatorname{det}\left(a_{i}^{j}\right)\right]
$$

and dividing by the relations in Theorem 2. Then Corollary 5 says that (for $n \geqslant 3$ ) there are nontrivial nilpotent elements in $A$. We can define a smooth subgroup 
$F_{\text {red }}(R)$ of $F(R)$ by imposing the further condition that all nilpotent expressions in $A$ vanish on the entries of elements in $F_{\text {red }}$ (When $R$ is a field, $F_{\text {red }}(R)=F(R)$.) We already have $\mathrm{GL}_{n}$ mapping into $F$ with kernel $\mu_{2}$, and hence the group scheme quotient $\mathrm{GL}_{n} / \mu_{2}$ is embedded as a closed subgroup of $F$. As $\mathrm{GL}_{n}$ is smooth, so is $\mathrm{GL}_{n} / \mu_{2}$, and thus $\mathrm{GL}_{n} / \mu_{2}$ is inside $F_{\text {red }}$. But $F_{\text {red }}$ is connected (since $F$ is), and by Proposition 4 it has the same dimension as $\mathrm{GL}_{n} / \mu_{2}$. By $[5,12.4]$, we have equality:

Proposition 6. We have $F_{\text {red }} \simeq \mathrm{GL}_{n} / \mu_{2}$.

If $R$ is reduced (i.e., without nilpotents), then any solution in $R$ of the equations defining $F$ will satisfy the further conditions defining $F_{\text {red }}$. Thus

COROllary 7. If $R$ is reduced, $F(R) \simeq\left(\mathrm{GL}_{n} / \mu_{2}\right)(R)$.

This corollary answers a question raised in [6], where the case $n=3$ was worked out. It does not imply that the map from $\mathrm{GL}_{n}(R)$ to $F(R)$ is surjective, since the map $\mathrm{GL}_{n} \rightarrow \mathrm{GL}_{n} / \mu_{2}$ is only a group scheme epimorphism. As shown in [6], the true conclusion is

Proposition 8. Suppose $R$ is reduced. Then the maps $\lambda P\left(\right.$ ) $P^{\text {tr }}$ ( for invertible scalar $\lambda$, matrix $P$ ) are a normal subgroup of $F(R)$. When $n$ is odd, they are all of $F(R)$. When $n$ is even, the quotient is isomorphic to the group of invertible $R$-modules $M$ satisfying $M \otimes M \simeq R$ and $\oplus_{1}^{n} M \simeq R^{n}$.

For $R$ containing nilpotents, this proposition will not hold. That is illustrated by the computation with $R=\mathbf{F}_{2}[\varepsilon]$ in Proposition 3 .

\section{Application to Jordan algebras.}

THEOREM 9. Let $k$ be a field. Let $A$ be a central simple algebra over $k$, and suppose $J$ is an involution of orthogonal type on $A$. Let $H(A, J)$ be the fixed set of $J, a$ (quadratic) Jordan algebra over $k$, and let $N$ be its generic norm polynomial. The only invertible linear maps $\varphi: H \rightarrow H$ for which $N(\varphi X)$ is a constant multiple of $N(X)$ are those of the form $\varphi(X)=\lambda c X c^{J}$, for invertible $\lambda$ in $k$ and $c$ in $A$.

ProOF. This result was proved by Jacobson [2, 3] except in characteristic 2, so we may assume $\operatorname{char}(k)=2$. (An argument like the one here would, of course, work also in other characteristics.) Let $F_{1}$ be the affine group scheme preserving the polynomial $N$. Let $G$ be the affine group scheme defined by $G(R)=(A \otimes R)^{\times}$. We have a group scheme homomorphism $G_{m} \times G \rightarrow F_{1}$ sending $(\lambda, c)$ to the map $X \mapsto \lambda c X c^{J}$. We want to show that $G_{m}(k) \times G(k)$ maps onto $F_{1}(k)$.

We know that over the algebraic closure $\bar{k}$ there is an isomorphism of $A \otimes \bar{k}$ to some $M_{n}(k)$ taking $J$ to the transposition map. Thus over $\bar{k}$ we see that the Jordan algebra $H$ becomes isomorphic to the symmetric matrices. Hence $N$ becomes the determinant, and $F_{1}$ becomes the $F$ of the previous section, while $G$ becomes $\mathrm{GL}_{n}$. In particular, this shows that $G_{m} \times G$ is smooth, since it is so after base extension. It follows that $G_{m} \times G$ has a smooth image $G_{1}$, a closed subgroup of $F_{1}$. Over $\bar{k}$, this $G_{1}$ becomes $\mathrm{GL}_{n} / \mu_{2}$, and is equal to $F_{\text {red }}$. 
The argument now is routine. Let $A_{1}$ be the ring representing $F_{1}$; over $\bar{k}$, we know that $A_{1} \otimes \bar{k}$ is isomorphic to $A \otimes \bar{k}$, where $A$ is the ring introduced just before Proposition 6. The closed subgroup $G_{1}$ of $F_{1}$ is represented by some quotient ring $A_{1} / I$. As $G_{1}$ becomes $F_{\text {red }}$ over $\bar{k}$, we see that

$$
\left(A_{1} / I\right) \otimes \bar{k}=(A \otimes \bar{k}) /(\text { nilpotents }) .
$$

Hence $I$ consists of nilpotents. Since the field $k$ is certainly reduced, it follows that all elements of $F_{1}(k)$ are actually in $G_{1}(k)$. Finally now we observe that $G_{m} \times G \rightarrow$ $G_{1}$ has kernel consisting of the $(\lambda, \alpha I)$ with $\lambda \alpha^{2}=1$, since this is true after we extend to $\bar{k}$. This kernel is isomorphic to $G_{m}$ under the map $(\lambda, \alpha I) \rightarrow \alpha$. But the cohomology group $H^{1}\left(\bar{k} / k, G_{m}\right)$ is trivial, and it follows that $\left(G_{m} \times G\right)(k) \rightarrow G_{1}(k)$ is surjective $[5,18.1]$.

Thus we see that the norm similarities here in characteristic 2 are the same as in other characteristics. But a related question brings out the special nature of characteristic 2 . The Lie algebra of the group scheme $F_{1}$ in this theorem is, in Jacobson's terms, the Lie algebra leaving the generic norm semi-invariant. In other characteristics, $F_{1}$ equals the structure group $G_{1}$, and so Lie $F_{1}$ is the same as the $n^{2}$-dimensional algebra Lie $G_{1}$. But in characteristic 2 , since $F_{1}$ becomes isomorphic to $F$ over $\bar{k}$, the dimension of Lie $F_{1}$ is given by Proposition 3, and for $n \geqslant 3$ this is much larger than $n^{2}$. In other words, though the norm similarities $F_{1}$ here differ only "infinitesimally" from the structure group $G_{1}$, the difference reveals itself in the Lie algebra preserving the norm.

\section{REFERENCES}

1. G. Frobenius, Über die Darstellung der endlichen Gruppen durch lineare Substitutionen. Berlin Sitzungsber, 1897, pp. 994-1015; Abhandlungen III, pp. 83-103.

2. N. Jacobson, Structure and representations of Jordan algebras, Amer. Math. Soc., Colloq. Publ., vol. 39. Amer. Math. Soc., Providence, R. I., 1968.

3. Structure groups and Lie algebras of Jordan algebras of symmetric elements of associative algebras with involution, Adv. in Math. 20 (1976), 106-150.

4. T. Muir, A treatise on the theory of determinants, Dover, New York, 1960.

5. W. C. Waterhouse, Introduction to affine group schemes, Graduate Texts in Math., No. 66, Springer-Verlag, New York, 1979.

6. _ A A _ Atomorphisms of $\operatorname{det}\left(X_{i j}\right)$ : The group scheme approach, Adv. in Math. (to appear)

7. _ Twisted forms of the determinant, J. Algebra 86 (1984), 60-75.

Department of Mathematics, Pennsylvania State University, University Park, Pennsylvania 16802 